\title{
Glioblastoma formation in a recurrent intracranial epidermoid cyst: a case report
}

\author{
Paul MacMahon ${ }^{1,2}$, Collin M Labak ${ }^{3}$, Sarah E Martin-Bach ${ }^{4,5}$, Ahmad Issawi ${ }^{1,2}$, Kiran \\ Velpula ${ }^{3}$ \& Andrew J Tsung*,1,2,3 \\ ${ }^{1}$ Department of Neurosurgery, University of Illinois College of Medicine at Peoria, Peoria, IL, 61605, USA \\ ${ }^{2}$ Neurosurgery Department, OSF HealthCare Illinois Neurological Institute, Peoria, IL, 61637, USA \\ ${ }^{3}$ Department of Cancer Biology \& Pharmacology, University of Illinois College of Medicine at Peoria, Peoria, IL, 61605, USA \\ ${ }^{4}$ Department of Pathology, University of Illinois College of Medicine at Peoria, Peoria, IL, USA \\ ${ }^{5}$ Neuropathology, OSF HealthCare Illinois Neurological Institute, Peoria, IL, 61637, USA \\ *Author for correspondence: Tel.: +1 309655 2700; Fax: +1 309655 7696; Andrew.J.Tsung@INI.ORG
}

\section{CNS Oncology}

\begin{abstract}
Background: Transformation to glioblastoma following recurrent epidermoid cyst resection has not been reported. Chronic inflammation can underlie malignant transformation of epidermoid cysts. Astrogliosis following repeated resections may have induced the rare transformation to glioblastoma. Clinical presentation: A patient presenting with left lower extremity weakness was found to harbor a parietal mass lesion. Histopathology demonstrated an epidermoid cyst. Following multiple re-resections, an intra-axial mass was discovered within the operative bed, confirmed as glioblastoma. Conclusion: This is the first report of glioblastoma associated with a resected epidermoid cyst. Subsequent to resection, the chronic inflammatory milieu propagated by astrogliosis is thought to have induced malignancy. The progression to glioblastoma draws attention to neoplastic transformation in the context of recurrent epidermoids.
\end{abstract}

First draft submitted: 14 February 2018; Accepted for publication: 30 August 2018; Published online: 13 December 2018

Keywords: epidermoid cyst • glioblastoma • malignant transformation

\section{Importance}

Intracranial epidermoid cysts (IEC) account for an estimated $1 \%$ of primary intracranial lesions and are considered pathologically benign. Nonetheless, the malignant transformation of IEC into squamous cell carcinoma (SCC) is a well-documented, albeit rare, occurrence [1]. The mechanism of such transformation has been theorized to be secondary to chronic inflammation in the region of the IEC, most commonly, secondary to cyst rupture. Given the cellular lineage, carcinogenesis of the encapsulated squamous epithelium, composing the IEC, into a squamous cell malignancy is relatively easily conceived. However, here we report a unique circumstance - a case of a glioblastoma (WHO grade IV) arising within the resection cavity of a recurrent IEC, following two previous resections. Though Poyuran et al. have previously reported the coexistence of chordoid glioma and epidermoid cyst, to the best of our knowledge our description of the development of a glioma in a resection bed not previously demonstrated to harbor glial atypia represents a unique occurrence, hitherto not definitively reported [2]. We utilize this novel case to propose mechanisms by which malignant transformation may occur given the unequivocal presentation and transition from benign to malignant pathology.

\section{Clinical presentation}

In October 2007, a 64-year-old Caucasian previously healthy female and with no significant family history of disease developed progressive dizziness, left lower extremity weakness, and gait disturbances. MRI demonstrated a $7.4 \mathrm{~cm}$ diffusion-restricting, cystic, non-enhancing extra-axial mass lesion centered along the medial right parietal lobe (Figure $1 \mathrm{~A} \& \mathrm{~B}$ ). Surgical resection was undertaken to the level of the splenium and within the confines of what was believed to represent appropriately restrained and a patient with high-preoperative neurological function. Nonetheless, near gross-total resection was achieved during surgery (Figure 1C \& D). Histopathology demonstrated findings consistent with an IEC (data not shown). Postoperatively, no adjuvant therapy was administered.

In December 2008, routine surveillance follow-up imaging demonstrated significant recurrence (Figure 2A \& 

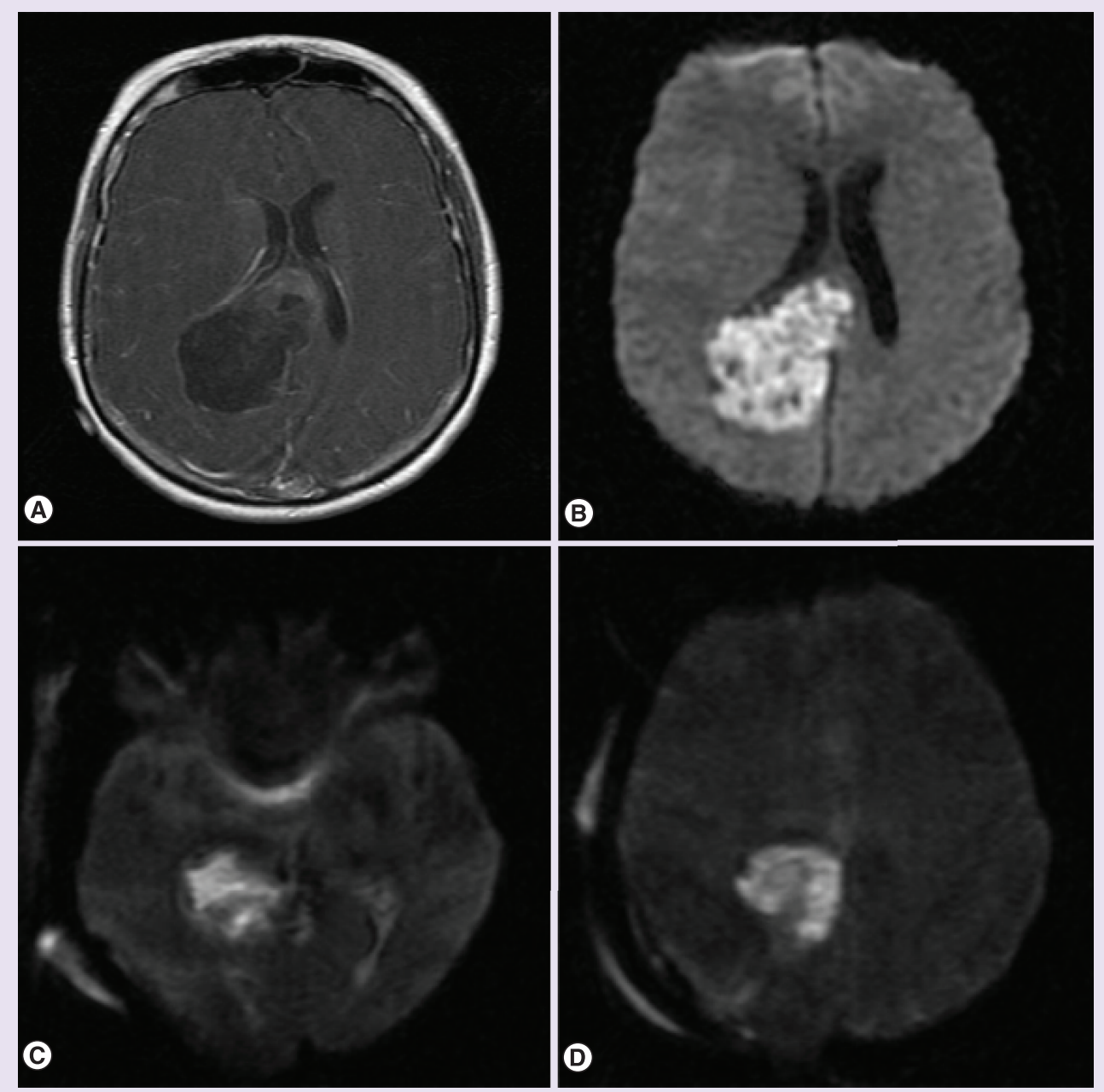

Figure 1. 2007 imaging. (A) 2007 preoperative contrast-enhanced MRI of the brain (coronal) showed a cystic, non-enhancing mass localized in the medial right parietal lobe and (B) DWI confirmed the diffusion-restricting, cystic lesion. (C \& D) Following surgical intervention, postoperative DWI showed partial resection of the lesion. DWI: Diffusion-weighted imaging.

B), prompting repeat right parietal craniotomies for re-resection (Figure 2C \& D). Histopathology demonstrated an epidermoid cyst without evidence of atypia or glial components (Figure 2E).

In March 2015, imaging tests again showed lesion recurrence (Figure 3A), confirmed by histopathologic examination. Right parietal craniotomy with re-resection was performed (Figure 3B \& C). Adjuvant radiotherapy was not performed following either resection.

In February 2016, routine ophthalmologic exam revealed asymptomatic left homonymous hemianopia. Further neurological examination revealed subtle left hemiparesis, in the absence of objective sensory changes. Repeat MRI demonstrated a new intra-axial mass within the splenium and periventricular white matter, along the previous operative bed, suggesting high-grade glioma (Figure 4A \& B). Gadolinium contrast media was contraindicated, due to the development of chronic renal failure. Repeat craniotomy was performed with intended maximal surgical resection, taken laterally to the level of the ventricle, with truncation of the mass at the level of the splenium (Figure 4C \& D). Histopathological analysis confirmed glioblastoma (GBM) diagnosis (WHO grade IV; Figure 4E).

The patient's postoperative course was uneventful, with stability of her pre-operative visual field deficits and hemiparesis. She was eventually discharged to a skilled nursing facility. Unfortunately, 4 weeks after discharge, the 

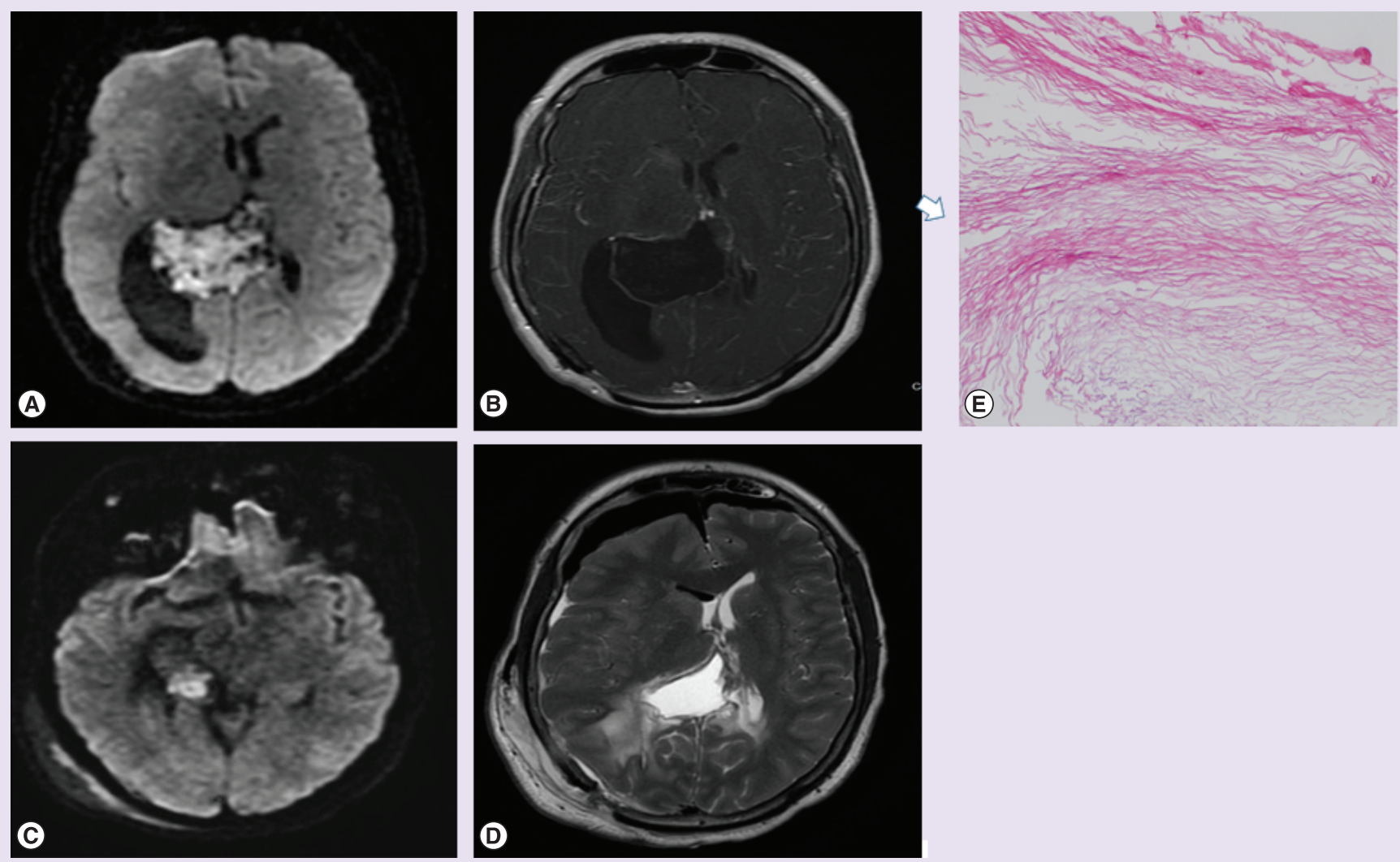

Figure 2. 2008 Imaging. (A) In December 2008, follow-up axial DWI and (B) preoperative MRI showed recurrence of resected parietal lesion. (C) Postoperative DWI and (D) axial T2-weighted MRI, obtained February 2009, demonstrates near total mass resection. (E) Microscopic examination of the patient's second resection specimen (February 2009) showed fragments of flaky keratinaceous debris, consistent with epidermoid cyst contents. No epithelial lining was identified in this specimen. No glial neoplasm was present (H\&E, 100X). DWI: Diffusion-weighted imaging; H\&E: Hematoxylin \& eosin.
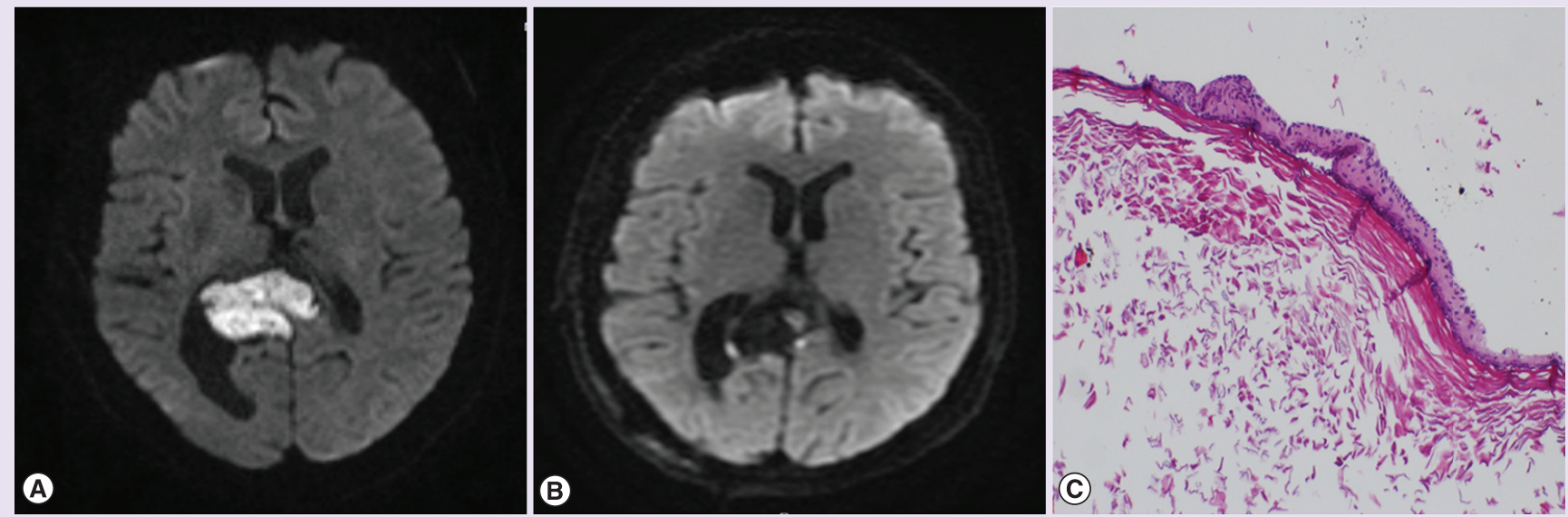

Figure 3. 2014 Imaging. (A) In December 2014, preoperative DWI of the brain (coronal) identified recurrence lesion, localized to the operative bed. (B) Postoperative brain DWI confirmed recurrence. (C) Microscopic examination of the patient's third resection specimen (March 2015) revealed benign stratified squamous epithelial cyst lining with flaky keratinaceous cyst contents, consistent with recurrent/residual epidermoid cyst. No glial neoplasm was present (H\&E, 100X).

DWI: Diffusion-weighted imaging; H\&E: Hemotoxylin and eosin. 

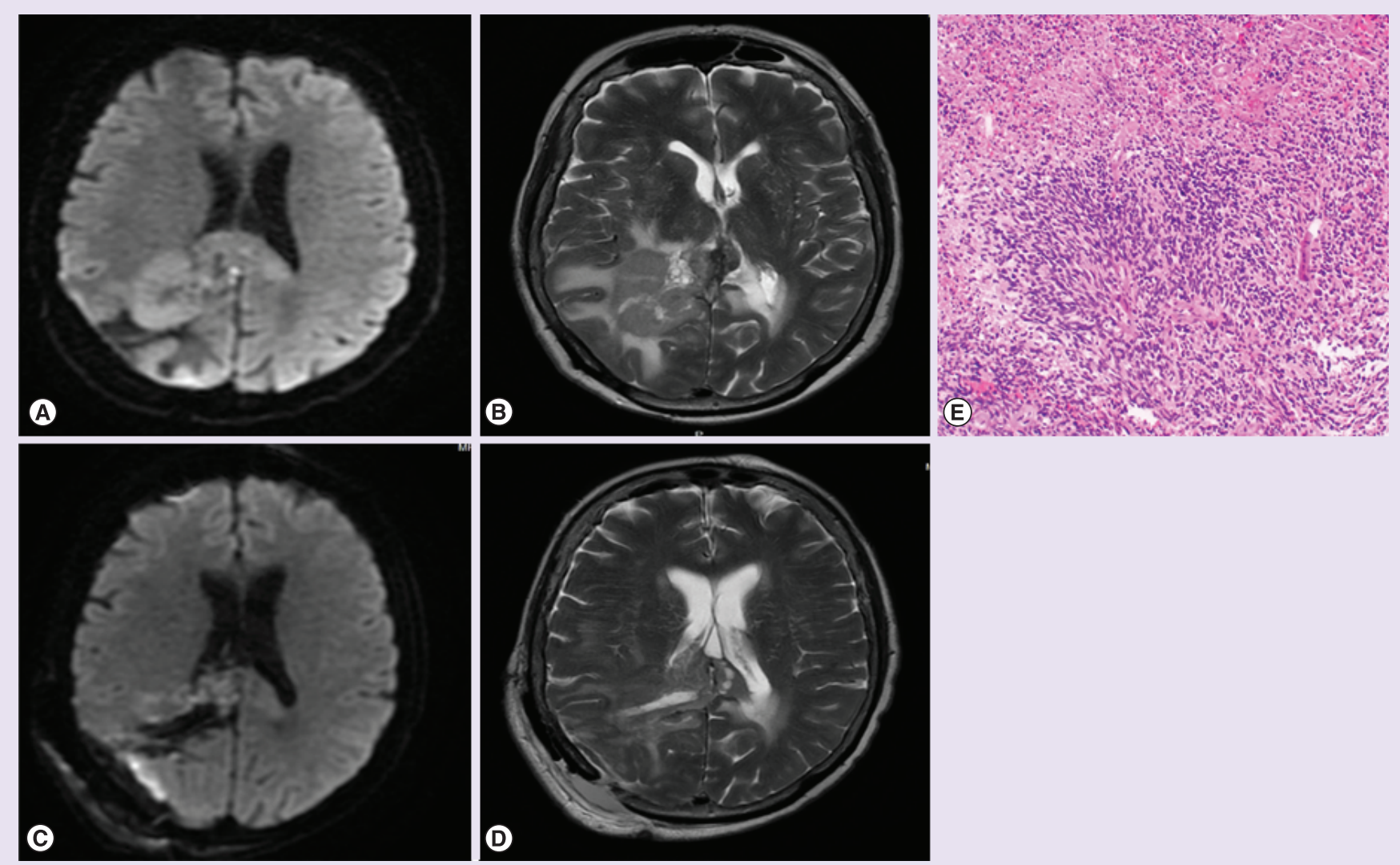

Figure 4. 2016 Imaging. (A) Preoperative DWI and (B) axial T2-weighted MRI, obtained February 2016, showed new lesion formation, intra-axial within the splenium and periventricular white matter, along the previous operative bed (no contrast, administered secondary to low GFR). (C) Postoperative DWI and (D) axial T2-weighted MRI showed near total lesion resection. (E) Microscopic examination of the patient's fourth and final resection specimen (February 2016) showed a high-grade infiltrating astrocytic neoplasm with areas of necrosis (top of image), consistent with glioblastoma. Areas of recurrent/residual epidermoid cyst were also seen (not shown; H\&E, 100X).

DWI: Diffusion-weighted imaging.

patient suffered a colonic perforation for which she underwent emergent intervention. During this hospital stay, she succumbed to complications related to acute pulmonary embolism.

Patient consent was not obtained as the patient expired.

\section{Discussion}

IECs are congenital lesions believed to develop within the 4th or 5th week of gestation, secondary to retention of ectodermal elements, thus resulting in inappropriate sequestration of squamous epithelium. With time, repetitive desquamation results in lesion enlargement. Despite this enlargement, IECs are considered benign, accounting for approximately $1 \%$ of intracranial lesions, commonly occurring within the cerebellopontine angle.

Despite their traditionally benign nature, transformation into malignancy, namely SCC, has been well documented. An exhaustive literature review by Nagasawa et al. uncovered 58 reported cases of such transformation, since 1912 [1]. Overall, the prognosis within this population starkly contrasted that of traditional IEC, with a mean survival of just under 1 year, regardless of adjuvant therapies rendered. The average time interval between initial diagnosis and transformation was found to be anywhere from 6 to 15 years.

The first reported case of transformation can be dated back to 1910, when Stromeyer reported a case of malignant changes within the epithelial lining of an IEC [3]. Interestingly, circumstances surrounding this case were met with contention regarding the exact nature of these reported changes. In 1955, Henschen addressed these controversies, hypothesizing that reported changes were potentially a combination of epidermoid and glioblastoma, 
citing knowledge of one other such case [4]. Since this original paper, however, the literature has been void of further reported instances of IEC occurring in close conjunction with high-grade glioma.

Epidermoid carcinogenesis secondary to chronic inflammation from either idiopathic/iatrogenic cyst rupture or the intra-operative deposition of foreign material has been commonly theorized. Unquestionably, inflammation does have a role in induction and maintenance of neoplastic growth. Mantovani et al. propose a model of converging cancer and inflammatory pathways in which NF-kB, HIF $1 \alpha$ and STAT3 collectively produce cytokines in stromal, tumor and inflammatory cells alike [5]. The product of this synergism is a chronic inflammatory microenvironment, which is highly conducive, if not necessary, for carcinogenesis. To substantiate this model, Giannoni et al. showed that cancer-associated fibroblasts utilize transcription factors NF- $\mathrm{KB}, \mathrm{HIF} 1 \alpha$ and their downstream product COX2 to induce the release of reactive oxygen species, thus giving rise to epithelial-mesenchymal shift (EMT) and subsequent stem cell formation [6]. Li et al. additionally identified IL-1 as a primary mediator of EMT and stem cell formation [7].

In our case, multiple surgical resections may well have resulted in a chronic inflammatory microenvironment, as astrogliosis and neural stem cell recruitment follows the trauma of surgery [8]. Additionally, the connection between inflammatory markers and glioblastoma formation is well substantiated. Puliyappadamba $e t$ al. noted the role of NF- $\kappa \mathrm{B}$ in glioma pathogenesis, citing the inflammation hallmark as a signaling target of mutant EGFRvIII and a mediator of glioblastoma proliferation, invasion and maintenance of GICs [9]. Strategies to inhibit NF$\kappa \mathrm{B}$, including the small molecule inhibitor DHMEQ, have seen some success in laboratory investigations, but remain in preclinical development [10]. Consistent with Mantovani's model, crosstalk between NF- $\kappa$ B and HIF $1 \alpha$ develops. Tafani et al. report on two conflicting papers, one indicating that NF-KB activates HIF $1 \alpha$, and another reporting the opposite [11]. In each of these papers, data supports that inflammation and the characteristic hypoxic microenvironment are interconnected elements that contribute to glioblastoma formation and maintenance.

An alternative, albeit far less likely, model of IEC-GBM transformation, involves de novo conversion of IEC to GBM. Acquisition of stemness would be a necessary prerequisite for epithelial to astrocytic cell lineage shift. Interestingly, certain genetic markers are shared between epithelial and astrocytic types, suggesting that molecular oncogenesis occurs by similar signaling cascades. For example, Oh et al. found cytokeratin immunoreactivity to be common to high-grade metastatic carcinoma and glioblastoma, with no marker demonstrating $100 \%$ specificity for metastatic carcinoma [12]. Another common marker, PIK3CA, was reported to be amplified in 11 of 73 primary tumors by Gallia et al. who used lung SCC as a control for PIK3CA amplification, confirming this characteristic amplification in some malignant carcinomas [13]. An additional shared factor common to the two neoplasms is HGF, which remains important to cell migration and invasion in both neoplasms. Abounader and Laterra report on the presence of HGF and its receptor tyrosine-protein kinase MET (c-Met) in brain tumor growth and angiogenesis, citing a mutation of this receptor-ligand complex in glioblastoma [14]. There is a robust body of literature regarding induction of the HGF/c-Met axis in carcinomas encompassing a variety of lineages, indicating that the axis is involved in EMT [15], evasion of apoptosis [16] and tumor progression [17].

\section{Conclusion}

This report illustrates a rare case of a non-SCC malignancy, secondary to IEC presence and/or resection factors. The patient's diagnosis of glioblastoma development within the IEC resection cavity likely represents de novo presentation of high-grade glioma. Carcinogenesis induced by chronic inflammation subsequent to numerous surgical resections is posited, with the low-probability de novo shift in cell type also a possibility. This case represents a unique entity affiliating glioblastoma with the setting of prior multiple IEC resections, in the absence of previous adjuvant therapies.

Financial \& competing interests disclosure

The authors have no relevant affiliations or financial involvement with any organization or entity with a financial interest in or financial conflict with the subject matter or materials discussed in the manuscript. This includes employment, consultancies, honoraria, stock ownership or options, expert testimony, grants or patents received or pending, or royalties.

No writing assistance was utilized in the production of this manuscript.

Ethical conduct of research

The authors state that they have obtained appropriate institutional review board approval or have followed the principles outlined in the Declaration of Helsinki for all human or animal experimental investigations. In addition, for investigations involving human subjects, informed consent has been obtained from the participants involved. 
Open access

This work is licensed under the Attribution-NonCommercial-NoDerivatives 4.0 Unported License. To view a copy of this license, visit http://creativecommons.org/licenses/by-nc-nd/4.0/

\section{Executive summary}

- Transformation to glioblastoma following recurrent epidermoid cyst resection has not been reported.

- This is the first report of glioblastoma associated with a resected epidermoid cyst.

- Intracranial epidermoid cysts (IEC) account for an estimated $1 \%$ of primary intracranial lesions and are considered pathologically benign.

- IECs are congenital lesions believed to develop within the 4th or 5th week of gestation, secondary to retention of ectodermal elements, thus resulting in inappropriate sequestration of squamous epithelium.

- In our case, multiple surgical resections may well have resulted in a chronic inflammatory microenvironment, as astrogliosis and neural stem recruitment follows the trauma of surgery.

- This report illustrates a rare case of a non-squamous cell carcinoma malignancy, secondary to IEC presence and/or resection factors.

- This case represents a unique entity affiliating glioblastoma with the setting of prior multiple IEC resections, in the absence of previous adjuvant therapies.

\section{References}

Papers of special note have been highlighted as: $\bullet$ of interest; $\bullet \bullet$ of considerable interest

1. Nagasawa D, Yew A, Spasic M, Choy W, Gopen Q, Yang I. Survival outcomes for radiotherapy treatment of epidermoid tumors with malignant transformation. J. Clin. Neurosci. 19, 21-26 (2012).

- First report of malignant transformation of intracranial epidermoid cyst.

2. Poyuran R, Mahadevan A, Sagar BK, Saini J, Srinivas D. Chordoid glioma of third ventricle with an epidermoid cyst: coexistence of common histogenesis? Int. J. Surg. Pathol. 24(7), 663-667 (2016).

3. Stromeyer F. Ober ein mit Sarkom kombiniertes Cholesteatom des Gehirns. Beitr. Path. Anat. 47, 392-398 (1910).

4. Henschen F. Handbuch der Speziellen Pathologischen Anatomie und Histologie, Band 13, Teil 3, Lubarsch O, Henke F, Rossle R (Eds), Springer Verlag, Berlin, p648 (1955).

5. Mantovani A, Allavena P, Sica A, Balkwill F. Cancer-related inflammation. Nature 454(7203), 436-444 (2008).

-. A review of pathological inflammation common to neoplasia.

6. Giannoni E, Bianchini F, Calorini L, Chiarugi P. Cancer associated fibroblasts exploit reactive oxygen species through a proinflammatory signature leading to epithelial mesenchymal transition and stemness. Antioxid. Redox Signal. 14(12), 2361-2371 (2011).

7. Li Y, Wang L, Pappan L, Galliher-Beckley A, Shi J. IL-1 $\beta$ promotes stemness and invasiveness of colon cancer cells through Zeb1 activation. Mol. Cancer 11, 87 (2012).

8. Fawcett JW, Asher RA. The glial scar and central nervous system repair. Brain Res. Bull. 49(6), 377-391 (1999).

9. Puliyappadamba VT, Hatanpaa KJ, Chakraborty S, Habib AA. The role of NF-kB in the pathogenesis of glioma. Mol. Cell. Oncol. 1(3), e963478 (2014).

10. Brassesco MS, Roberto GM, Morales AG et al. Inhibition of NF- $\kappa$ B by dehydroxymethylepoxyquinomicin suppresses invasion and synergistically potentiates temozolomide and $\gamma$-radiation cytotoxicity in glioblastoma cells. Chemother. Res. Pract. 2013, 593020 (2013).

11. Tafani M, Di Vito M, Frati A et al. Pro-inflammatory gene expression in solid glioblastoma microenvironment and in hypoxic stem cells from human glioblastoma. J. Neuroinflamm. 8, 32 (2011).

12. Oh D, Prayson RA. Evaluation of epithelial and keratin markers in glioblastoma multiforme: an immunohistochemical study. Arch. Pathol. Lab. Med. 123(10), 917-920 (1999).

13. Gallia GL, Rand V, Siu IM et al. PIK3CA gene mutations in pediatric and adult glioblastoma multiforme. Mol. Cancer Res. 4(10), 709-714 (2006).

14. Abounader R, Laterra J. Scatter factor/hepatocyte growth factor in brain tumor growth and angiogenesis. Neuro. Onc. 7(4), 436-451 (2005).

15. Mo CJ. Changes of glycan profiling in cell surface after hepatocyte growth factor (HGF) induced epithelial mesenchymal transition (EMT) in Huh7 hepatocellular carcinoma cells. Fud. Univ. Journ. Med. Sci. 41(2), 198-204 (2004).

16. Bu R, Uddin S, Bavi P et al. HGF/c-Met pathway has a prominent role in mediating antiapoptotic signals through AKT in epithelial ovarian carcinoma. Lab. Investig. 91(1), 124-137 (2011).

17. Casbas-Hernandez P, D’Arcy M, Roman-Perez E et al. .. Role of HGF in epithelial-stromal cell interactions during progression from benign breast disease to ductal carcinoma in situ.. Breast Cancer Res. 15(5), R82 (2013). 
